\title{
Benchmarking ist ein wirksames Instrument zur Verbesserung der klinischen Versorgung - Pro
}

\author{
Benchmarking is an Effective Instrument to Improve Clinical Services - Pro
}

Pro

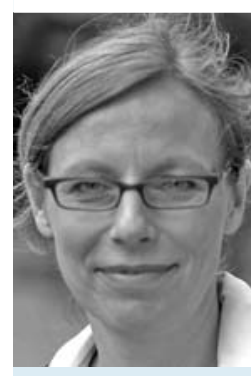

Birgit Janssen
Die Methode des

Benchmarkings stellt für die psychiatrischpsychotherapeutische Diagnostik, Behandlung und Versorgung ein relativ neues Verfahren dar. Grundlage des Benchmarking-Ansatzes ist es, relevante Dimensionen des Behandlungsprozesses und der Ergebnisbewertung mittels Kennzahlen oder Indikatoren zu erfassen und damit einer statistischen Auswertung und Analyse zugänglich zu machen. Die Indikatoren bilden eine Grundlage für den Vergleich unterschiedlicher Einrichtungen mit dem Ziel, „gute Praxis“ zu identifizieren und einem Erfahrungstransfer zugänglich zu machen. In diesem Sinne ist Benchmarking „Lernen von guten Ideen und Lösungen“ auf der Basis von systematischen Prozess- und Ergebnisvergleichen. „Benchmarking als Methode der Organisationsentwicklung bedeutet, besser werden durch Lernen von anderen“" [1]. Es ist kein starres Modell, in dem eine Institution „besser“ oder „schlechter“ ist, sondern beinhaltet eine kontinuierliche Veränderung.

Benchmarking in Psychiatrie und Psychotherapie wird leider häufig ausgesprochen kritisch gesehen. Der Begriff „Benchmarking“ impliziert möglicherweise $\mathrm{Zu}$ sammenhänge mit externem „Kostendruck“ oder mangelnder Individualität der Behandlung psychisch Kranker. Auf der anderen Seite wird in somatischen Bereichen der Medizin Benchmarking von Behandlungsprozessen und -ergebnissen in der Regel gut akzeptiert. Krankenhausvergleiche über den Anteil der Patienten mit nosokomialen Infektionen gehören inzwischen zur Routine und sind als Qualitätsstandard aus der medizinischen Routineversorgung nicht mehr wegzudenken. Benchmarking in der Medizin bedeutet nämlich mitnichten einem Kostendruck nachzugeben oder eine Behandlung und Versorgung zu entindividualisieren, sondern im Gegenteil, Benchmarking stellt datenbasiert sicher, dass Qualitätsstandards eingehalten und Potenziale der Qualitätsoptimierung ausgeschöpft werden, auch wenn dies mit höheren Kosten verbunden sein sollte.

Auch in der psychiatrisch-psychotherapeutischen Diagnostik, Behandlung und Versorgung gibt es inzwischen evidenzbasierte Qualitätsstandards. Dazu zählen nicht nur die in zahlreichen randomisierten Effektivitätsstudien untersuchten psychiatrischen und psychotherapeutischen Behandlungsverfahren, sondern insbesondere auch die mit Methoden der Versorgungsforschung untersuchten Elemente einer optimalen, ggf. sektorenübergreifenden, Behandlung und Versorgung auch und vor allem schwer psychisch kranker Menschen (z.B. [2]). Auf Basis dieser wissenschaftlichen Untersuchungen wurden auch für das psychiatrisch-psychotherapeutische Fachgebiet inzwischen eine Vielzahl evidenzbasierter Leitlinien entwickelt [3, 4]. Diese sind Grundlage für die Entwicklung evidenzbasierter Qualitätsindikatoren [5]. Qualitätsindikatoren sollten relevante Qualitätsaspekte der Versorgung genau und zuverlässig messen und in der Versorgungspraxis gut einsetzbar sein. Sie können als Struktur-, Prozess- und Ergebnisindikatoren abgeleitet werden und bilden die Basis eines Benchmarkings.

Benchmarking von Parametern der Strukturqualität birgt zwar nur selten eine konkrete Möglichkeit zur Optimierung, da diese Parameter häufig nicht direkt beeinflussbar sind, es ist jedoch Grundlage zur Beurteilung der Vergleichbarkeit und ist damit die Basis für eine möglicherweise notwendige Risiko- oder Case-mix-Adjustierung.

Ein Benchmarking von evidenzbasierten Indikatoren zur Prozessqualität kann dazu führen, dass einzelne Institutionen, zum Beispiel Krankenhäuser, aber auch regionale Versorgungsverbünde, eigene
Qualitätsdefizite der Behandlung, die der Leitlinienempfehlung nicht entsprechen, erkennen. So kann eine Optimierung der eigenen Behandlungsprozesse zur Verbesserung der Leitlinienadhärenz in Gang gesetzt werden. Insbesondere Ansätze von Benchmarking in einem „offenen“ Verbund von Kliniken oder ambulanten Leistungserbringern führen optimaler Weise dazu, dass durch kommunikative Prozesse zwischen den Teilnehmern die Implementierung von verbesserten Behandlungsprozessen erleichtert wird [6]. Ohne datenbasierte Messungen anhand evidenzbasierter Qualitätsindikatoren bleiben womöglich gut gemeinte Verbesserungen der Behandlungsprozesse beliebig und deren möglicher Einfluss auf den Behandlungserfolg lässt sich nicht adäquat abbilden. Natürlich müssen auch innovative Prozesse in die psychiatrisch-psychotherapeutischen Behandlung implementiert werden, für die möglicherweise aus unterschiedlichen Gründen (noch) keine evidenzbasierten Prozessindikatoren entwickelbar sind. Nur so ist Fortschritt in der Routineversorgung, also auch außerhalb von effektivitätsgeleiteten Studien, im psychiatrisch-psychotherapeutischer Behandlungsprozess möglich. Jedoch müssen sich auch gerade solche Innovationen der Routineversorgung einer kritischen Ergebnisdiskussion stellen.

Hierzu ist der Einsatz eines Benchmarkings von Ergebnisindikatoren sinnvoll. Das erwünschte Ergebnis einer Behandlung in der Medizin, auch in der Psychiatrie und Psychotherapie, ist ein möglichst hohes Maß an Gesundheit und sozialer Funktionsfähigkeit. Auch neue Behandlungsprozesse müssen sich hinsichtlich des Ergebnisses mit bereits bewährten Behandlungsprozessen messen lassen. Die Patienten interessiert primär das Ergebnis der Behandlung, das Benchmarking der Prozessindikatoren dagegen ist insbesondere hilfreich zur internen Qualitätsoptimierung.

Indikatoren zur Messung der Ergebnisqualität sind in der internationalen Litera- 
tur gut etabliert [7], allerdings finden sich für den deutschsprachigen Raum bisher wenig evidenzbasierte Ergebnisindikatoren, die institutionsübergreifend erhoben werden, sodass Benchmarking von Ergebnisindikatoren bisher einer individuellen Absprache zwischen Institutionen bedarf. Sollte der Prozess der Entwicklung von Qualitätsindikatoren durch den Gemeinsamen Bundesausschuss dazu führen, dass auch Ergebnisindikatoren gesetzlich vorgeschrieben erhoben werden müssen, dann könnte sich das in Zukunft ändern. Allerdings wird dies meines Erachtens nur dann zielführend sein, wenn Ergebnisindikatoren klinisch relevante Endpunkte der Behandlung messen und nicht nur einzelne Elemente des Behandlungsprozesses (wie z.B. Pharmakotherapie oder Zwangsbehandlungen) abbilden. Ergebnisindikatoren müssen durch Routinedaten oder einen minimalen Dokumentationsaufwand erhebbar sein. Diese Forderung erfüllen die in Deutschland bisher entwickelten Qualitätsindikatoren nur zu einem geringen Teil [8]. Jedoch gibt es erste Erfolg versprechende Ansätze aus dem angloamerikanischen Raum [9] und der Schweiz [10], die zu einem regelmäßigen Benchmark von klinisch relevanten Ergebnisindikatoren psychiatrisch-psychotherapeutischer Behandlung genutzt werden. in Deutschland könnten Elemente der bisherigen „Cording-BADO“ gekürzt, aufwandsarm reaktiviert und hinsichtlich klinisch relevanter Endpunkte angepasst werden und würden so eine bundesweite gemeinsame Datenbasis für ein Benchmarking darstellen.

Aus den oben genannten Gründen der Behandlungsoptimierung sollte auf ein Benchmarking auch in der Psychiatrie und Psychotherapie nicht verzichtet werden. Viele Studien konnten zeigen, dass es durch Benchmarking zu einer erheblichen Optimierung der Behandlungsqualität und der Leitlinienkonformität (z.B. [6]) kam. Benchmarking als Unterstützung zur Sicherstellung einer adäquaten, sicheren, evidenzbasierten und vor allem an einem guten Ergebnis orientierten klinischen Behandlung sollte unseren Patienten nicht vorenthalten werden.

\section{Literatur}

1 de Cruppé W, Blumenstock G, Fischer I et al. Results of the evaluation of German benchmarking networks funded by the Ministry of Health. Z Evid Fortbild Qual Gesundhwes 2011; 105: 339-342

2 Gaebel W, Großimlinghaus I, Heun $R$ et al. European Psychiatric Association (EPA) guidance on quality assurance in mental healthcare. Eur Psychiatry 2015; 30: 360 387

3 Hasan A, Wobrock T, Gaebel W et al. National and international schizophrenia guidelines. Update 2013 regarding recommendations about antipsychotic pharmacotherapy. Nervenarzt 2013; 84: 1359-1360, 1362-1364, 1366-1368. Review. German

4 Gühne $U$, Weinmann S, Arnold $K$ et al. S3 guideline on psychosocial therapies in severe mental illness: evidence and recommendations. Eur Arch Psychiatry Clin Neurosci 2015; 265: $173-188$
5 Großimlinghaus I, Falkai P, Gaebel $W$ et al. Developmental process of DGPPN quality indicators. Nervenarzt 2013; 84: 350-365

6 Janssen B, van Brederode M, Kitzig $F$ et al. Benchmarking in psychiatric acute care - a demonstration project in a hospitals network. Z Evid Fortbild Qual Gesundhwes 2011; 105: 371-375

7 Spaeth-Rublee B, Pincus HA, Huynh PT. IIMHL Clinical Leaders Group, Mental Health Quality Indicator Project Measuring quality of mental health care: a review of initiatives and programs in selected countries. Can J Psychiatry 2010; 55: 539-548

8 Großimlinghaus I, Falkai P, Gaebel $W$ et al. LVR-Klinikverbund Assessment of quality indicators with routine data: Presentation of a feasibility test in ten specialist clinics for psychiatry and psychotherapy. Nervenarzt 2015; 86: 1393-1399

9 Glied SA, Stein BD, McGuire TG et al. Measuring Performance in Psychiatry: A Call to Action. Psychiatr Serv 2015; 66: 872-878

10 http://www.anq.ch/psychiatrie/

Sie haben eine eigene Meinung zu diesem Thema? Dann schreiben Sie uns an: psychiat-praxis@thieme.de!

Korrespondenzadresse Dr. med. Birgit Janssen

LVR Klinikum Düsseldorf, Klinik und Poliklinik für Psychiatrie und Psychotherapie Bergische Landstraße 2

40629 Düsseldorf

birgit.janssen@|vr.de

Bibliografie

Dol http://dx.doi.org/

10.1055/s-0041-111038

Psychiat Prax 2016; 43: 71-72

(c) Georg Thieme Verlag KG

Stuttgart · New York

ISSN 0303-4259 